\title{
Improved signal processing technique leads to more robust Self Diagnostic Accelerometer System
}

\author{
Roger Tokars ${ }^{1}$ and John Lekki ${ }^{2}$ \\ NASA Glenn Research Center, Brook Park, Oh, \\ 44135 \\ Dave Jaros $^{3}$ and M. Terrence Riggs ${ }^{4}$ \\ Apollo Research Corp, Buffalo, NY, 14225 \\ Kenneth P. Evans ${ }^{5}$ \\ Consultant Engineer, Cincinnati, Oh, 45244
}

\begin{abstract}
[Abstract] The self diagnostic accelerometer (SDA) is a sensor system designed to actively monitor the health of an accelerometer. In this case an accelerometer is considered healthy if it can be determined that it is operating correctly and its measurements may be relied upon. The SDA system accomplishes this by actively monitoring the accelerometer for a variety of failure conditions including accelerometer structural damage, an electrical open circuit, and most importantly accelerometer detachment. In recent testing of the SDA system in emulated engine operating conditions it has been found that a more robust signal processing technique was necessary. An improved accelerometer diagnostic technique and test results of the SDA system utilizing this technique are presented here. Furthermore, the real time, autonomous capability of the SDA system to concurrently compensate for effects from real operating conditions such as temperature changes and mechanical noise, while monitoring the condition of the accelerometer health and attachment, will be demonstrated.
\end{abstract}

${ }^{1}$ Electronics Engineer, Optical Instrumentation and NDE branch, 21000 Brookpark Rd./Mail Stop 77-1, AIAA Member.

${ }^{2}$ Electronics Engineer, Optical Instrumentation and NDE branch, 21000 Brookpark Rd./Mail Stop 77-1,

AIAA Member

3 Applications Engineer, Apollo Research Corp., 2300 Walden Ave. Buffalo, NY 14225.

${ }^{4}$ Director Research \& Engineering., Apollo Research Corp., 2300 Walden Ave. Buffalo, NY 14225.

${ }^{5}$ Consultant.

\section{Introduction}

A ccelerometers are commonly used for aircraft Aand spacecraft health monitoring ${ }^{1,2}$. An accelerometer measures acceleration levels that can be used to identify faults on the structure the accelerometer is mounted to. The accuracy of such a sensor is of critical importance in systems which use accelerometer data to take corrective action, such as an automated shut down of an engine. To ensure reliability of the accelerometer output, not only must the accelerometer's mechanical and electrical operation be verified, but also the mounting of the accelerometer must be guaranteed so that engine vibrations are transferred. Mounting techniques to ensure accelerometer attachment include safety wire, studs, glues, beeswax, magnets, and other mounting bases. Although erroneous data from the accelerometer may imply a lack of attachment, the investigator still may not know the true state of the accelerometer unless he can visually or remotely inspect the attachment. This may be difficult or impossible in some cases based on the scenario and location. The self diagnostic accelerometer (SDA) attempts to address this shortfall by continuously polling for accelerometer health and attachment.

The SDA system polls for accelerometer health and attachment by sending a frequency swept sinusoidal signal to its piezoelectric crystal and concurrently monitoring the accelerometer's frequency response, or diagnostic signal. The diagnostic signal contains details on the accelerometer that correlate to the following failure modes: a physically damaged accelerometer, electrical disconnection, and accelerometer detachment/loosening from the structure $^{3-9}$. The frequency swept sinusoidal signal is of low voltage to reduce the amount of electric fatigue, or degradation that a higher amplitude signal can cause to the accelerometer's ceramic crystal. ${ }^{10-14}$ The lower voltage signal is associated with a lower signal to noise ratio which can be a problem in the case of significant mechanical noise in the system, as is often the case for vibration sensing accelerometers. To address this issue, the SDA system applies the use of a new signal processing technique as well as signal averaging to increase the signal to noise ratio.

Previous work with the SDA system has shown that changing the accelerometer temperature results in a bias shift and/or resonant frequency shift in the 
diagnostic response ${ }^{9}$. The shift in the diagnostic response due to temperature directly conflicts with the SDA system's ability to correlate accelerometer mounting torque and attachment to resonant frequency shift. In order to compensate for the effects of temperature, the new SDA signal processing technique is developed and discussed in detail in this paper.

The prototype SDA system, shown in Figure 1, uses simple electronics in order to better meet the requirements for in-flight sensor testing. Compared to a commercial signal analyzer the SDA system is more portable, smaller, uses less power, and easier to use as it is tailored specifically to monitor the health and attachment of an accelerometer.

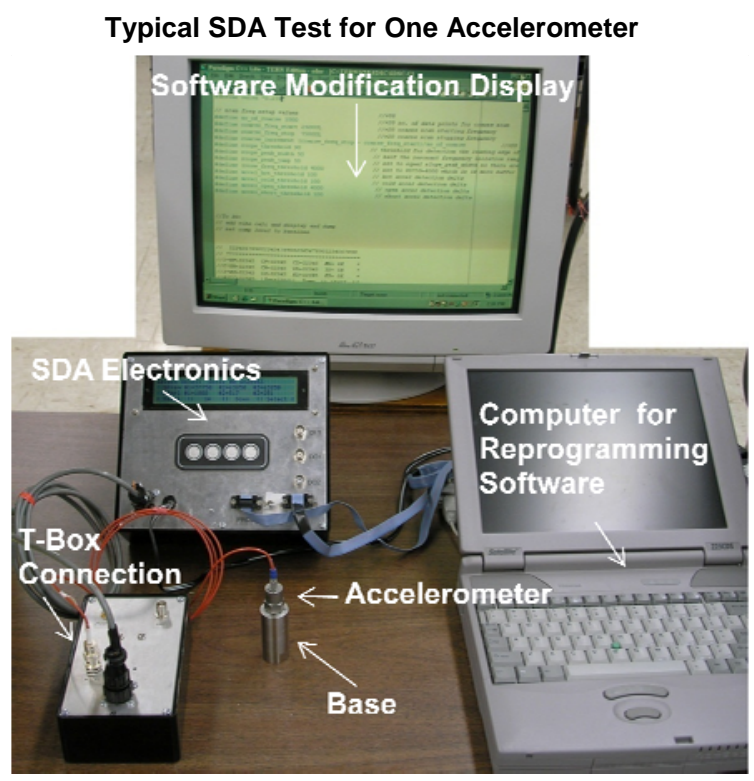

Figure 1. SDA test system demonstrating the various connections and controls.

\section{SDA System Software Development}

A. System Signal Processing Overview One of the critical goals of the SDA system is to detect accelerometer health and attachment. An attached condition is defined as an accelerometer having an attached torque of anything greater than or equal to 10 in-lbs. Previous SDA signal processing attempted to detect accelerometer health and attachment by simply monitoring the diagnostic response's resonant frequency amplitude, but the reliability of the technique came into question when compensating for the effects of temperature change. Temperature change shifted the diagnostic signal bias which often led to erroneous results in determining the accelerometer health and attachment. The new
SDA software with additional signal processing presented in this report attempts to address the issue of temperature bias shift as well as noise issues. Essentially, the new SDA software detects accelerometer health and attachment by monitoring for significant changes in the diagnostic response's resonant frequencies. The resonant frequencies are localized by finding the peaks of the differentiated signal response. By taking the derivative of the signal response, signal bias shifts are removed which simplifies detection of multiple resonant frequency peaks. Further noise reduction can be accomplished with signal averaging when necessary.

The SDA system concurrently measures both acceleration and diagnostic data, as shown in Figure 2. The SDA system does not interfere with the normal operation of the accelerometer because acceleration is generally measured in a lower frequency band, which can be up to $20 \mathrm{kHz}$, than that of the diagnostic data, which uses the $25 \mathrm{kHz}$ to 75 $\mathrm{kHz}$ high frequency range.

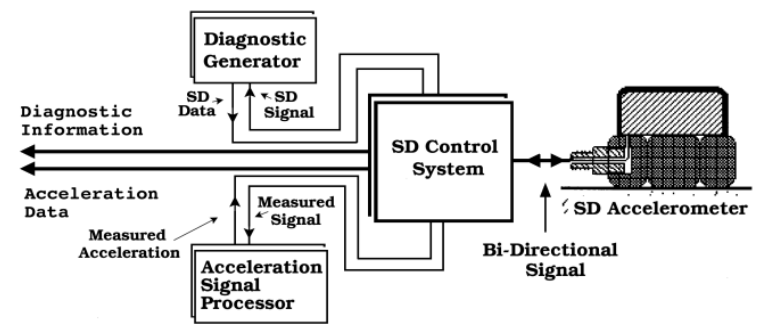

Figure 2. SDA system concurrently measures both acceleration and diagnostic data.

Because the SDA operates outside the normal operating frequency range of the accelerometer, the SDA system can be simply added on to most accelerometers as an added capability for monitoring accelerometer health and attachment. Once the SDA hardware is added, a baseline diagnostic response from the accelerometer is captured and saved at normal operating torque, temperature, and noise. The baseline diagnostic response not only captures the internal resonances of the accelerometer, but these resonances are also influenced by the impedance of the attached structure as well. Then during health and attachment monitoring of the SDA, this baseline diagnostic signal is repeatedly compared to the current diagnostic signal, as shown in Figure 3. More specifically, when the current resonant frequencies shift outside the normal operating range of the baseline resonant frequencies then a fault condition is triggered. 


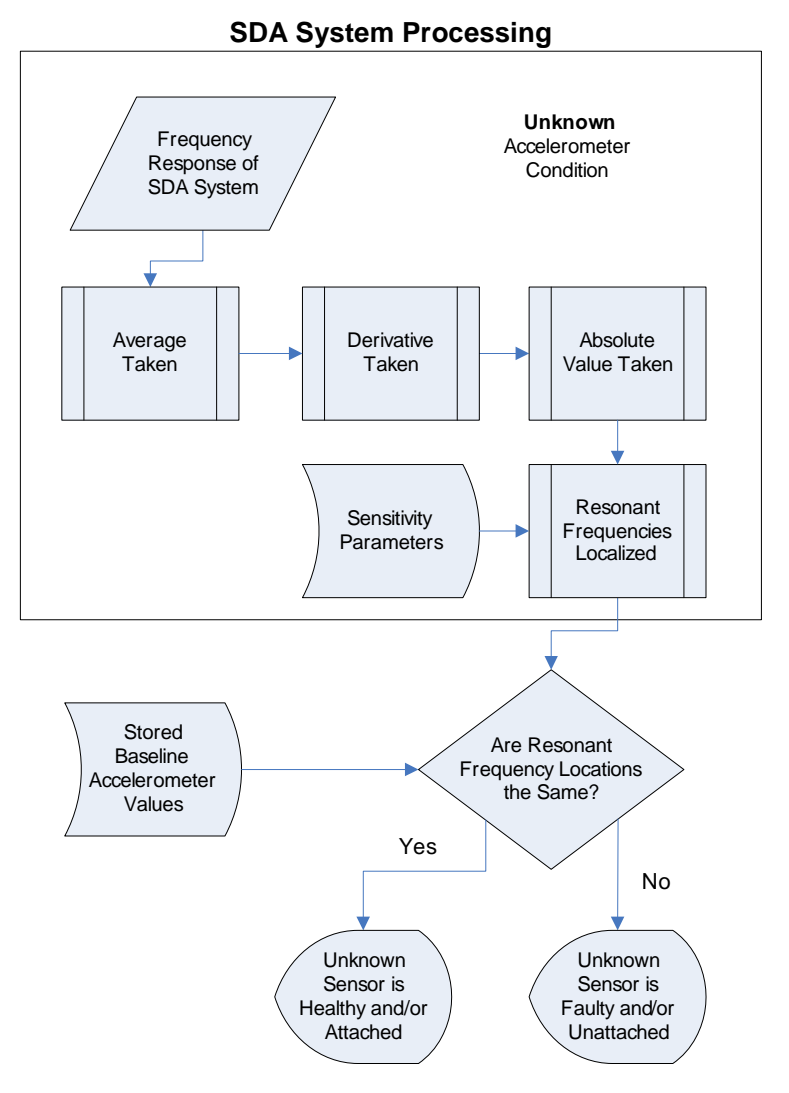

Figure 3. Flow chart of the SDA system signal processing. The boxed in Unknown Accelerometer Condition is the current operating condition of the accelerometer. The resonant frequencies from the current accelerometer are compared to the stored baseline resonant frequencies in order to determine accelerometer health and attachment.

The simplified hardware used in the SDA generates a comparable diagnostic signal to one generated by a commercial signal analyzer. Figure 4 shows the diagnostic signal, normalized to its relative minimum and maximum, for both the SDA system and a commercial signal analyzer for an attached accelerometer. The attached accelerometer diagnostic response is used as a baseline. Changes in the health and attachment of the accelerometer cause frequency shifts of the resonance peaks away from the frequencies stored in the attached/healthy baseline. Monitoring these changes is the goal of the new software technique.

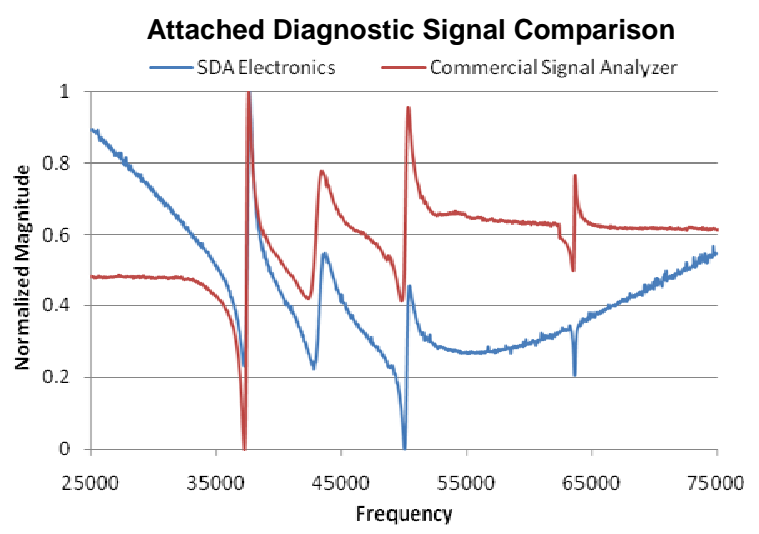

Figure 4. Diagnostic signal comparing the SDA electronics and a commercial signal analyzer for accelerometer \#0 attached at 30 in-lbs torque at room temperature.

The new SDA signal processing described in this report takes the derivative of the diagnostic signal. This cleans up the diagnostic signal so that important features, such as the resonant frequencies stand out. Figure 5 shows the absolute value of the derivative, normalized to its maximum, of the diagnostic signal in Figure 4. It can be shown from these figures that the identification of resonant frequencies can be made through an amplitude threshold detection of the data in Figure 5, while this is not the case for the data in Figure 4.

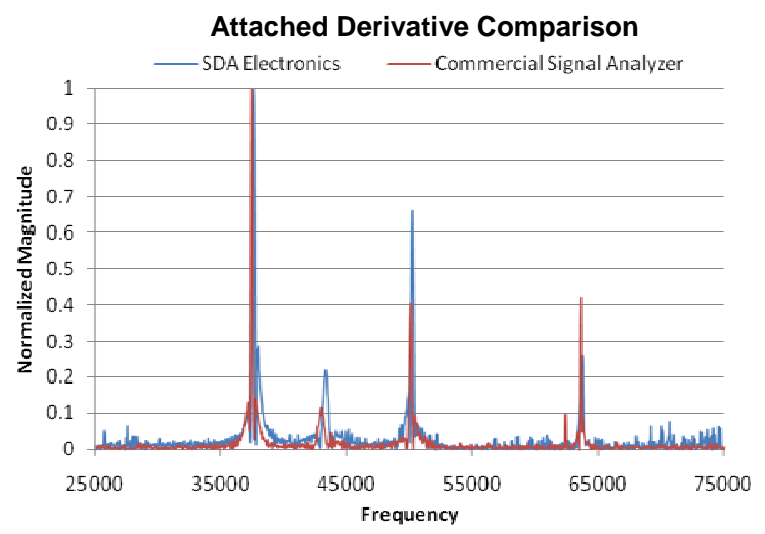

Figure 5. Absolute value derivative of the previous diagnostic signal comparing the SDA electronics and a commercial signal analyzer for accelerometer \#0 attached at 30 in-lbs torque at room temperature.

The signal response of the same accelerometer untorqued to the quarter loose position is shown in Figure 6. The signal response is normalized to the attached minimum and maximum (data in Figure 4). The associated derivative, normalized to the attached derivative maximum (data in Figure 5), is shown in Figure 7. 


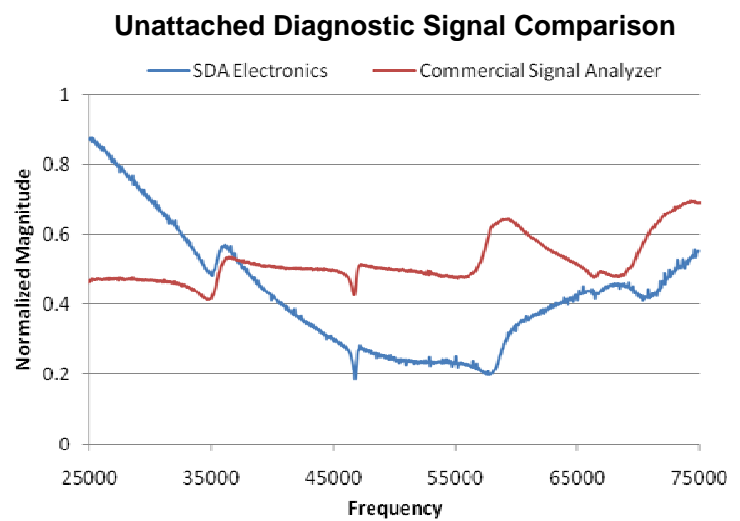

Figure 6. Diagnostic signal comparing the SDA electronics and a commercial signal analyzer for accelerometer \#0 unattached at quarter turn loose at room temperature.

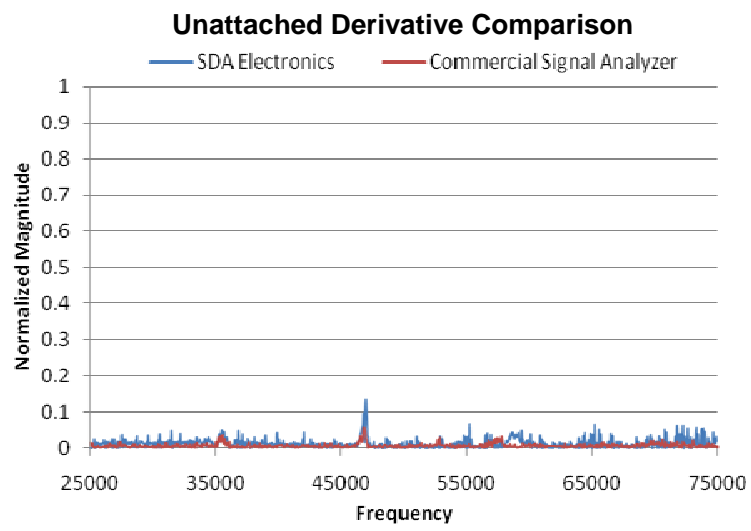

Figure 7. Absolute value derivative of the previous diagnostic signal comparing the SDA electronics and a commercial signal analyzer for accelerometer \#0 unattached at quarter turn loose at room temperature.

The important conclusions from Figures 4-7 are: the SDA electronics/system and the commercial signal analyzer plots agree with one another in terms of the frequencies where the accelerometer response is resonant. The derivative of the frequency response data simplifies the identification of particular resonant frequencies which is the essential information in the diagnostic response. Lastly, there is a noticeable change in the signal from the attached condition, Figure 5, to the unattached condition, Figure 7.

\section{B. Assessment Parameters}

Several assessment parameters are used by the SDA software for successful signal processing. The most important assessment parameters include averaging range, derivative threshold, and resonant frequency correlation range. The assessment parameters together determine the amount the current diagnostic signal is allowed to change from the baseline diagnostic signal before triggering a fault. Although the SDA system in this report was optimized for a specific accelerometer, the flexibility of the software and designated assessment parameters allows for customization to the desired electromechanical properties of any chosen accelerometer.

\section{Averaging Range}

The averaging range reduces the effects of noise generated on the diagnostic signal response by taking an average across a designated frequency range of data points. This moving average has the effect of smoothing out the signal response and any noise that may be in the signal. Figure 8 shows an example signal response comparing the use of 20 point averaging range for a 40 inch-lbs torque attachment with noise generated by a shaker system $^{15}$. The plotted frequency range has a resonant frequency in the middle of the figure.

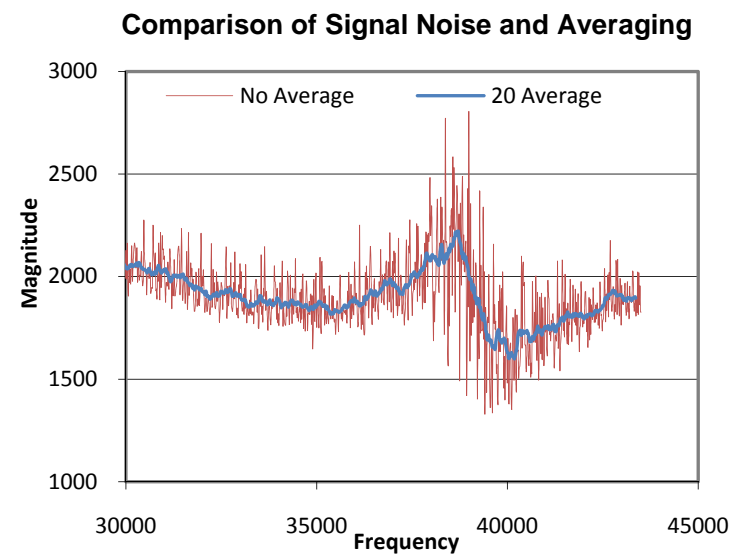

Figure 8. A resonant frequency diagnostic signal with noise caused from a shaker acceleration at $3.6 \mathrm{~g}$ maximum and the corrective averaging range across 20 data points, or approximately 300 hertz.

\section{Derivative Threshold}

As previously discussed, the SDA system takes the derivative with respect to frequency of the signal response to determine the resonant frequency locations. Figure 9 plots the signal response and its resulting derivative for an accelerometer with several different attachment torques. The resonant frequency locations are identified by determining if the magnitude of the derivative amplitude crosses a predefined threshold, called the derivative threshold. In the case of Figure 9, resonant frequencies for the attached condition would be located at approximately $37.5 \mathrm{kHz}$ and $50.5 \mathrm{kHz}$ for derivative threshold 1. If only one resonant frequency was desired to be tracked, the derivative threshold 2 could be used, which has a resonant frequency at approximately $37.5 \mathrm{kHz}$. 


\section{Signal Response and Resulting Derivative}

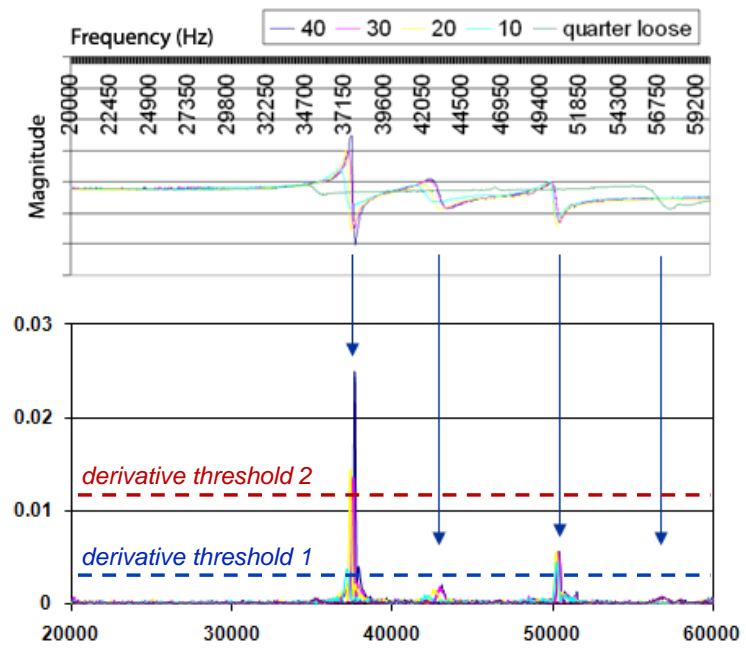

Figure 9. The signal response and its corresponding absolute value derivative for an accelerometer at 40, 30, 20, 10 inch-lbs torque and quarter loose.

Selecting the correct averaging range and derivative threshold is crucial in determining the resonant frequency locations. The derivative threshold is determined by inspecting the baseline signal derivative of the SDA system in the attached condition. The threshold should be low enough so that the resonant frequency is above the threshold while being high enough so that it is above signal noise. The threshold must also compensate for the smoothing effect of the averaging range which corresponds to a reduction in the resonant frequency derivative peak. Experimental tests in this report concurrently optimize the averaging range and derivative threshold for the SDA system in several different noise environments.

\section{Resonant Frequency Correlation Range}

The resonant frequency correlation range is the range that determines whether two resonant frequencies, each from a different signal response, are close enough to be considered matching resonances. Matched and unmatched resonances are used by the SDA algorithm to determine the attached and unattached fault conditions of the SDA system as shown in the Flow Chart of Figure 3.

\section{Other Assessment Parameters}

Other assessment parameters were included to aid in the software's flexibility and reduce possible error. To reduce the effect of noise spikes, a counter variable allowed the user to determine the minimum number of data points that had to be above the derivative threshold for resonant frequency determination. Two other SDA assessment parameters determine the high and low signal response magnitude limits which correlate to an open and short circuit respectively.

\section{Processed Output}

The real time SDA software algorithm was designed and tested with post processing software before being installed into the prototype SDA system. Figure 10 shows successful post processing software results for an attached condition with optimized assessment parameters.

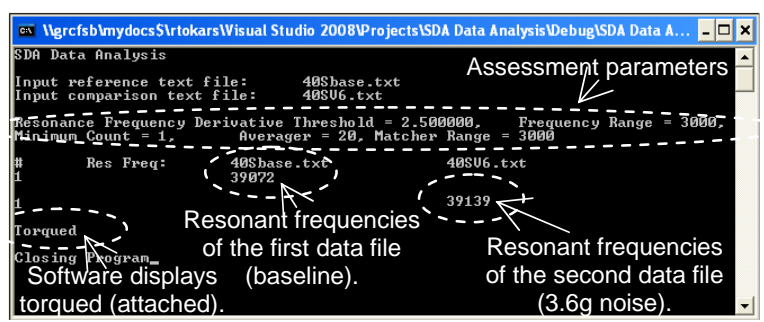

Figure 10. The SDA post processing software shows the matched resonant frequencies and correctly displays that the accelerometer is attached.

Once all baseline and assessment parameters were defined, the software was then downloaded into the SDA system to demonstrate real time autonomous inspection. For easy viewing, the SDA system outputs the accelerometer health and attachment information onto an LCD screen shown in Figure 11. Navigation through the screen by the four available buttons allows for other SDA system functions such as saving the inspected signal response to file. The saved file records a frequency vs. signal response as well as other SDA processed values and parameters. This file is useful for post processing analysis and accelerometer baseline determination.

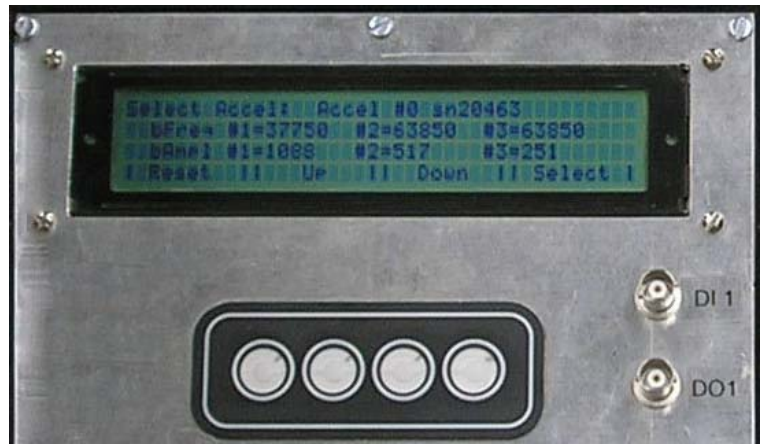

Figure 11. The SDA system LCD screen displays the results of real time inspection. The second row displays two resonant frequency locations, labeled \#1 and \#2. The four circular buttons are used to navigate through the screen as well as to perform various functions such as saving the signal response from the accelerometer. 


\section{Experimental Procedure}

Torque, noise, and temperature testing of the SDA system was repeated multiple times across ten accelerometers, labeled \#0-9. The purpose of using multiple accelerometers was to investigate the SDA system's ability to accommodate for variation in the assembly impedance in each accelerometer, specifically concerning the differences in the internal resonant frequencies. To accommodate for these differences, the SDA system requires a reliable and flexible processing technique to monitor the resonant frequencies while also taking into account the effects of noise and temperature change. The experimental test results show consistent and successful SDA system determination of accelerometer health and attachment.

\section{A. Torque Investigation}

A typical accelerometer is attached to a structure with a screw to ensure undamped transfer of vibrations from the structure. Using a torque wrench the accelerometer is attached to an exact torque level.

The three objectives of the Torque Investigation were to characterize the signal response resonant frequencies, to investigate the unattached and attached accelerometer condition, and to calibrate the SDA system to compensate for the frequency shift caused by changes in the torque of an attached accelerometer. Investigating the unattached and attached accelerometer condition involved gradually increasing the torque of an unattached accelerometer to the attached condition. Calibration of the SDA system involved optimizing the resonant frequency correlation range to successfully match similar signal response resonant frequencies. It is described further in the Analysis and Discussion section.

The SDA system was tested with each accelerometer attached with varying levels of torque. Multiple repetitions of testing were conducted with ten accelerometers for each torque condition of $1 / 4$ turn loose, 10 in-lbs, 20 in-lbs, 30 in-lbs, and 40 in-lbs at a specified constant temperature.

Another test condition was done at room temperature to observe the effects of gradually increasing the torque from a loose condition, to just touching, to tighter, and then finally to maximum finger tightness, estimated at 2-4 in-lbs torque. The data was collected using both a commercial signal analyzer and a prototype SDA microprocessor-based electronics unit. The test setups for the commercial signal analyzer and the SDA electronics are shown in Figures 12 and 13 respectively. The two setups also used a switchbox, as shown in Figure 14. The switchbox allowed the systems to easily swap between multiple accelerometers during a given test run.

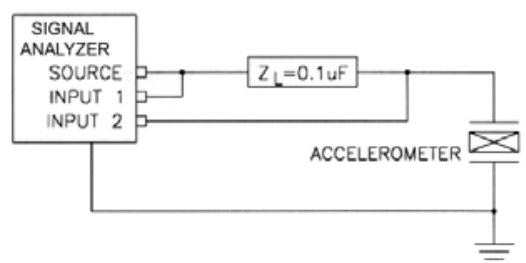

Figure 12. Test setup with accelerometer connected to the commercial signal analyzer.

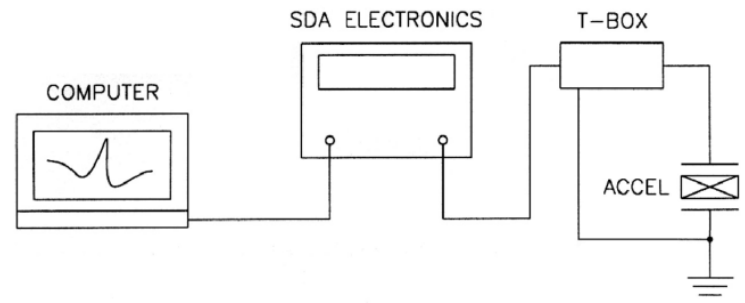

Figure 13. Test setup with accelerometer connected to the SDA microprocessor-based electronics.

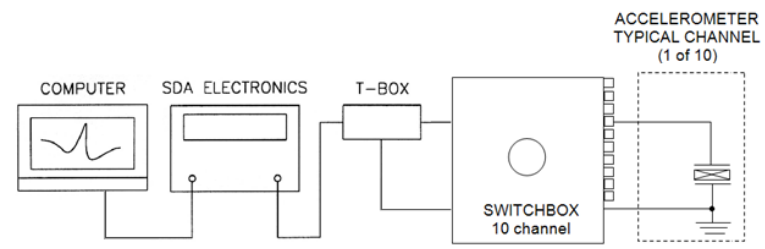

Figure 14. Test setup with multiple accelerometers and switchbox connected to the SDA microprocessor-based electronics.

\section{B. Vibration Investigation}

An accelerometer attached to an engine undergoes varying amounts of vibration during normal operation. If these vibrations are of a frequency in the diagnostic band, they show up as noise in the diagnostic signal response. To understand how the mechanical vibrations of the accelerometer will affect the diagnostic signal, a detailed study of the SDA signal response as the accelerometer experiences varying amounts of noise was conducted. A shaker system was used to simulate the vibrations coming from an engine.

The objective of the Vibration Investigation was to calibrate the SDA system to compensate for noise in the signal response. Calibration of the SDA system involved monitoring the SDA signal response under various levels of shaker noise. A three point averaging range was used to optimize the derivative threshold to successfully localize the signal response resonant frequencies. 
Noise was introduced into the SDA system with the use of a shaker system, shown in Figure 15 and 16, to simulate vibrations such as those experienced during typical operating environments. The vibrations cause amplitude jumps in the diagnostic signal response which can result in erroneous triggering of a fault condition. A commercial signal analyzer was programmed to create periodic chirp or random noise with a designated frequency bandwidth. Low or high levels of noise were generated with the shaker amplifier by changing the voltage to 1 or 5 volts respectively. A $10 \mathrm{mV} / \mathrm{g}$ reference accelerometer with a relatively flat high frequency response was used to monitor the mechanical excitation to the test accelerometers.

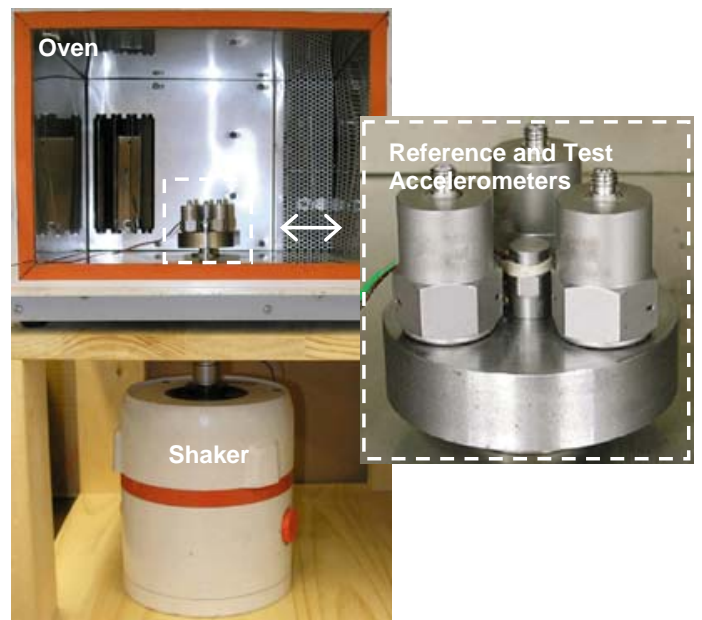

Figure 15. Electromechanical shaker setup with attached base connected to four accelerometers inside a temperature controlled oven.

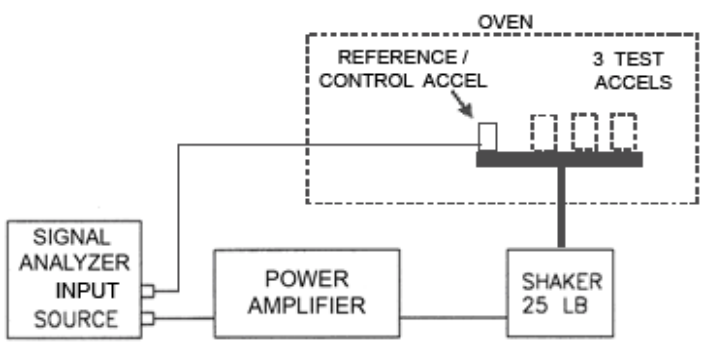

Figure 16. Electromechanical shaker system setup. One reference control accelerometer monitors the acceleration experienced by the other three test accelerometers.

\section{Temperature Investigation}

A typical accelerometer may experience a range of temperatures during normal operating conditions. An oven and/or liquid nitrogen bath were used to simulate these conditions.

The objective of the Temperature Investigation was to calibrate the SDA system to compensate for frequency shift caused by change in temperature. Calibration of the SDA system involved optimizing the resonant frequency correlation range to successfully match similar signal response resonant frequencies. Optimizing the resonant frequency correlation range is described further in the Analysis and Discussion section.

A thermocouple was used to determine when the target temperature of the accelerometers and mounting bases was reached. An additional base was manufactured to accept the thermocouple. Since this base has the same physical size as the ten mounting bases, it is assumed to have the same heat soak and heat sink characteristics. The thermocouple and ten accelerometers are shown in Figure 17.

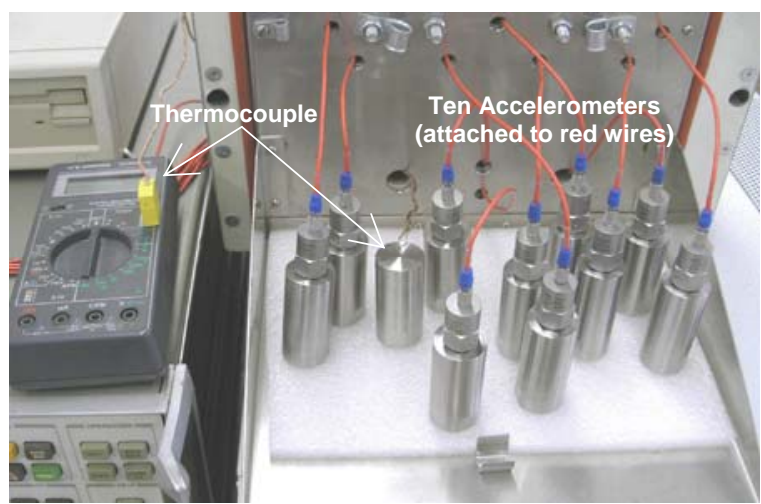

Figure 17. The temperature test setup with ten accelerometers and thermocouple before being put inside the oven.

The range of temperatures at which the accelerometers were tested included $-73^{\circ} \mathrm{C},-50^{\circ} \mathrm{C}$, $25^{\circ} \mathrm{C}$, room temperature (approximately $+25^{\circ} \mathrm{C}$ ), $+75^{\circ} \mathrm{C}$, and $100^{\circ} \mathrm{C}$. Multiple repetitions of testing were conducted with ten accelerometers for each torque condition of $1 / 4$ turn loose, 10 in-lbs, 20 in-lbs, 30 in-lbs, and 40 in-lbs at each temperature. The data was collected using both a commercial signal analyzer and the prototype SDA system. As previously noted, the test setups for the signal analyzer, the SDA electronics, and the switchbox are shown in Figure 12, 13, and 14 respectively. 
D. Real Time Vibration and Temperature Tests

The objective of the Vibration and Temperature Tests is to determine if the calibrated SDA system could successfully determine an attached and unattached condition while compensating for various levels of noise and temperature in real time. Using the setup and assessment parameters determined in the Torque, Vibration, and Temperature Investigations, the SDA system was extensively tested with ten accelerometers to determine SDA consistency and reliability. A detailed test matrix was used to test various shaker noise levels, torque levels ( $1 / 4$ turn loose to 30 inch-lbs), and temperatures $\left(-73^{\circ} \mathrm{C}\right.$ to $\left.+100^{\circ} \mathrm{C}\right)$. The test setup is consistent with previous test setups shown in Figures 13-17.

During the real time operation tests, the SDA system was also tested for the correct identification of open and short circuit faults. This additional health monitoring capability of the SDA system simulates a complete failure of the accelerometer's signal path. The open and short circuit are accomplished by disconnecting and grounding the accelerometer cable respectively.

\section{Experimental Results}

\section{A. Torque Investigation}

Characterization of the SDA signal response was done to localize distinct resonant frequencies. Figure 18 shows the typical signal response with labeled resonant frequency peaks for ten attached accelerometers for the same torque and temperature. As a comparison, Figure 19 shows the same ten accelerometers in the unattached $1 / 4$ turn loose condition.

\section{Ten Attached Accelerometers}

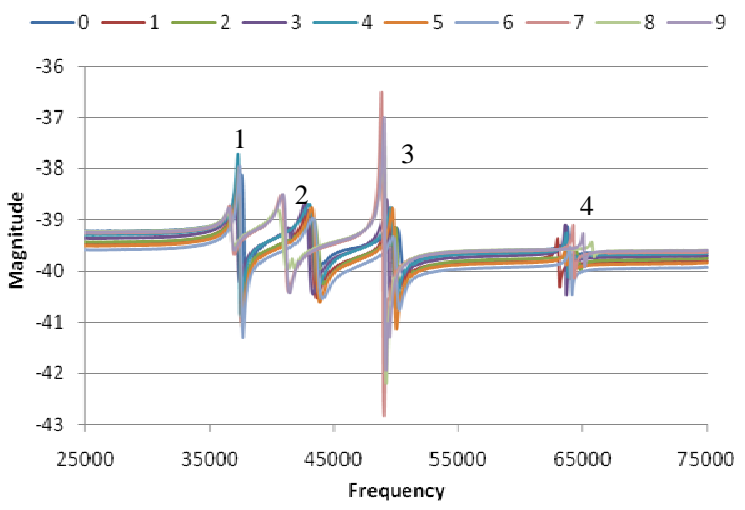

Figure 18. Signal response of ten accelerometers attached at 30 in-lbs torque at $0^{\circ} \mathrm{C}$ temperature. Four resonant frequency locations are numbered on the plot.
Ten Unattached Accelerometers

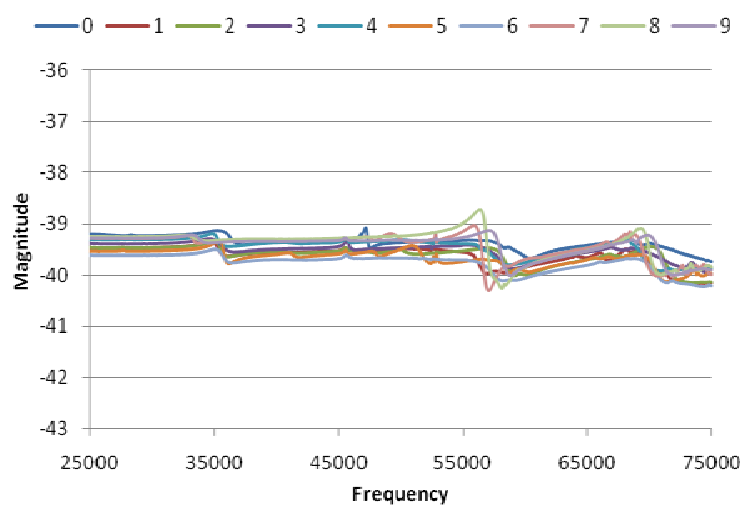

Figure 19. Signal response of ten accelerometers unattached at $1 / 4$ turn loose at $0^{\circ} \mathrm{C}$ temperature. Notice that the resonant frequencies are not easily recognizable.

Investigating the unattached and attached condition involved gradually increasing the torque incrementally and recording the signal response change. The results for the ten accelerometers tested were similar to those for Accelerometer \#0 shown in Figure 20.

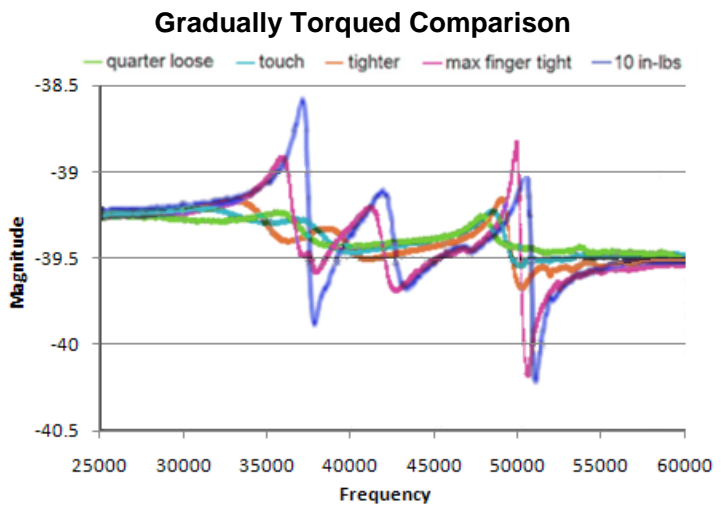

Figure 20. Signal response of Accelerometer \#0 as it is gradually increased in torque from quarter loose to 10 in-lbs torque. During the gradual increase in torque, the resonant frequencies shifted and became larger in amplitude in a gradual continuous motion.

Calibration of the SDA system was done to compensate for frequency shift caused by changes in the torque of an attached accelerometer. The largest frequency shift occurred between the two attached torque extremes, with 40 in-lbs being the tightest and 10 in-lbs being the loosest. Figure 21 shows the corresponding frequency shifts for some of these extremes. The largest frequency shift was approximately $500 \mathrm{~Hz}$. 
Accelerometer \#0 Torque Comparison

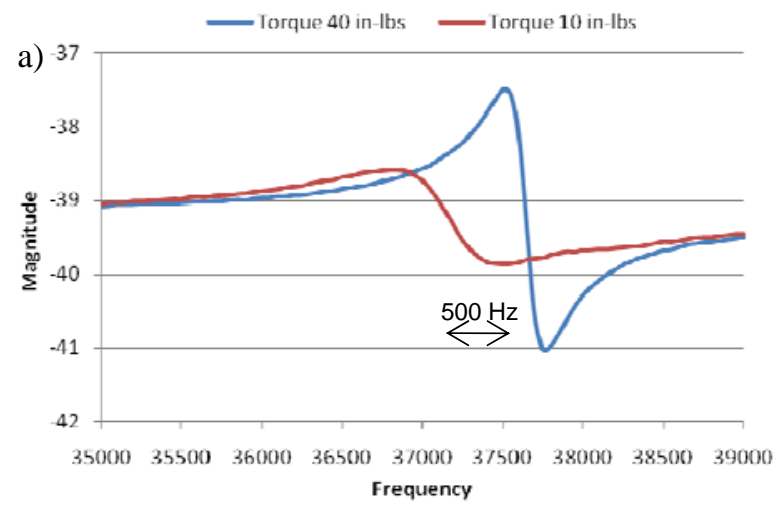

Accelerometer \#2 Torque Comparison

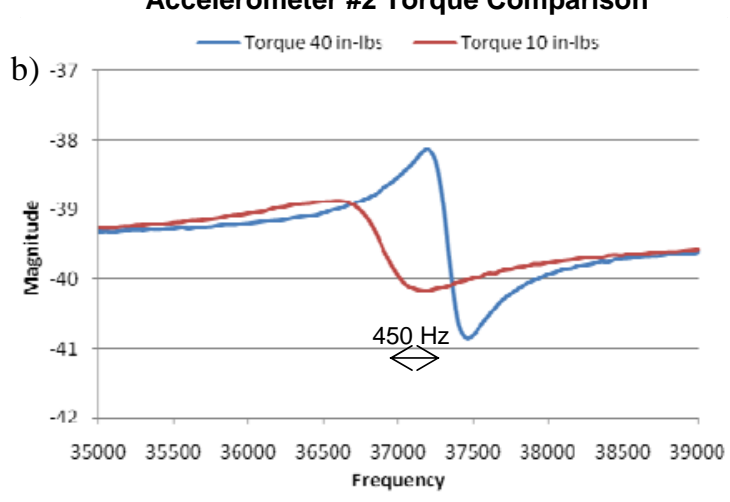

Accelerometer \#8 Torque Comparison

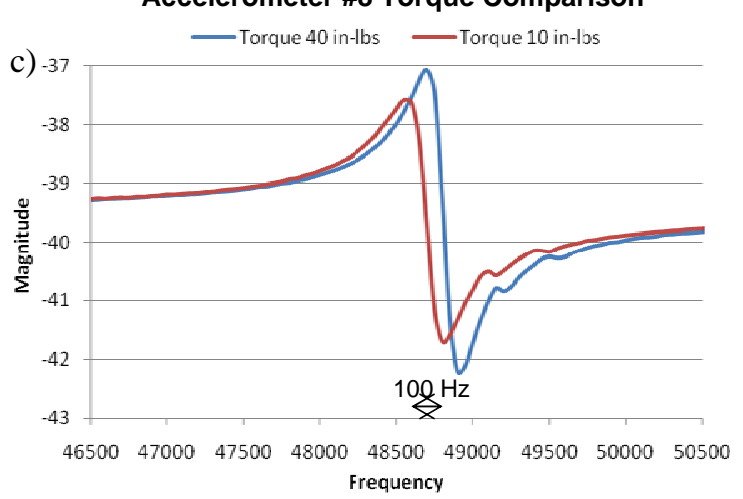

Figure 21. Resonant frequency shift from torque change of 40 in-lbs to 10 in-lbs in room temperature for Accelerometer a) \#0, $500 \mathrm{~Hz} \mathrm{b)} \mathrm{\# 2,} 450 \mathrm{~Hz} \mathrm{c}$ ) \#8, $100 \mathrm{~Hz}$

\section{B. Vibration Investigation}

The SDA system responses for various shaker noise tests are shown as follows. They include the attached conditions for periodic chirp and random noise. The associated reference accelerometer with calculated acceleration in g's is also attached to the figures. The loose condition was not plotted because it did not show any noise.
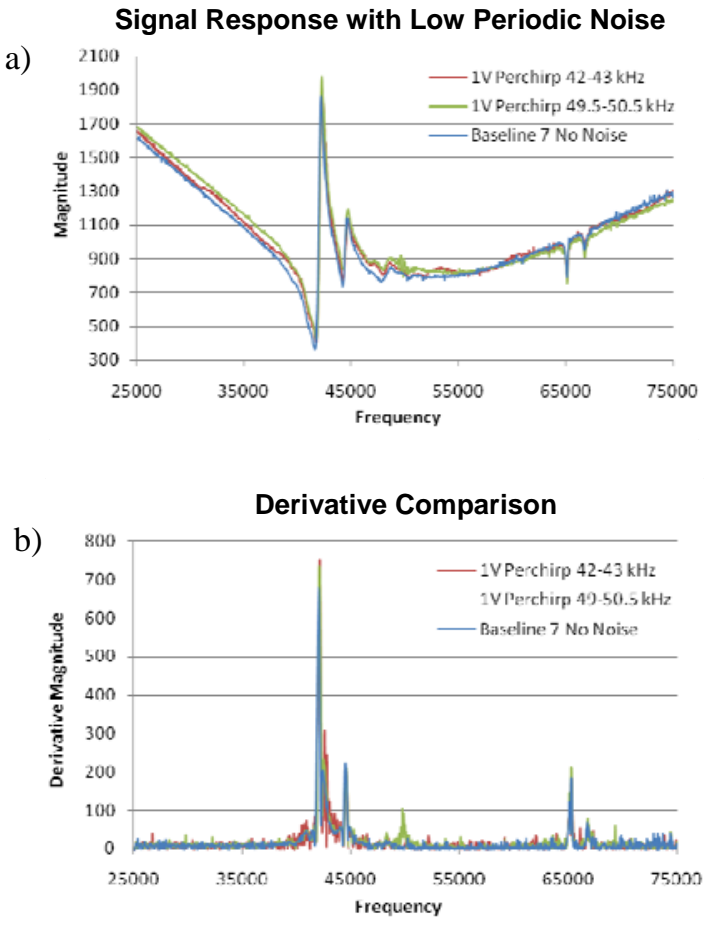

Reference 1V Periodic 42-43 kHz

c)

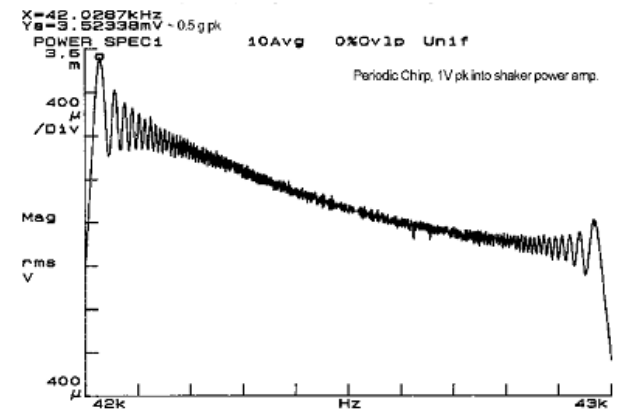

d)

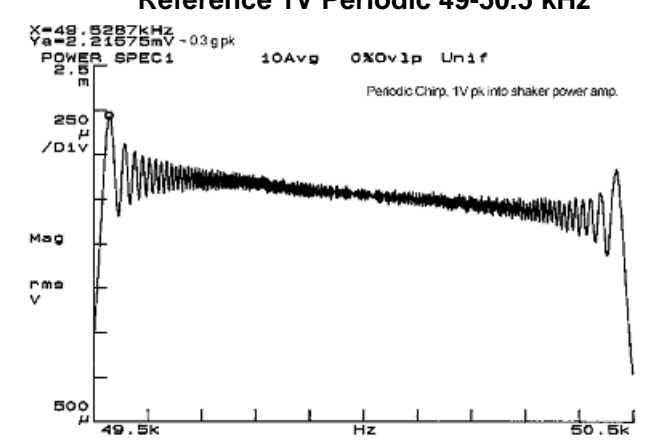

Figure 22. a) Signal response comparing baseline signal to two low periodic chirp noise cases for Accelerometer \#7 attached at 30 in-lbs torque at room temperature. b) Absolute value derivative of the signal responses. c) Noise band with peak acceleration at approximately $0.5 \mathrm{~g}$ and d) $0.3 \mathrm{~g}$. 
Signal Response with High Periodic Noise

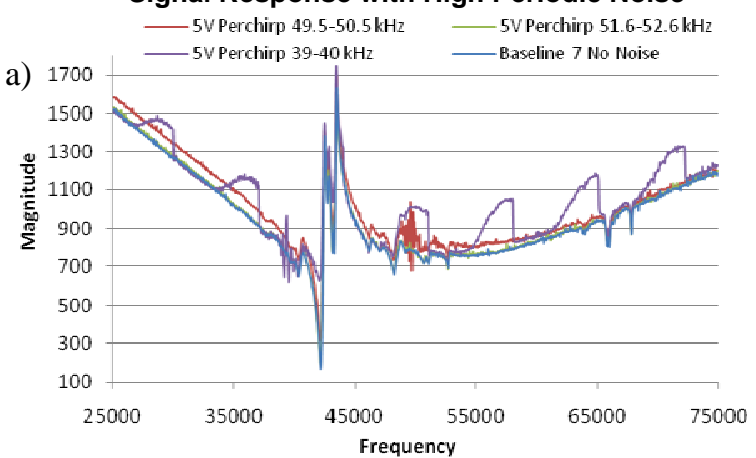

\section{Derivative Comparison}

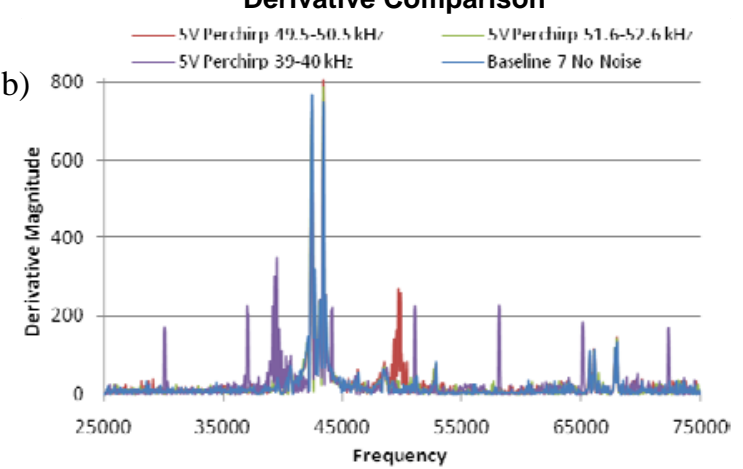

Reference 5V Periodic 49.5-50.5 kHz

c)

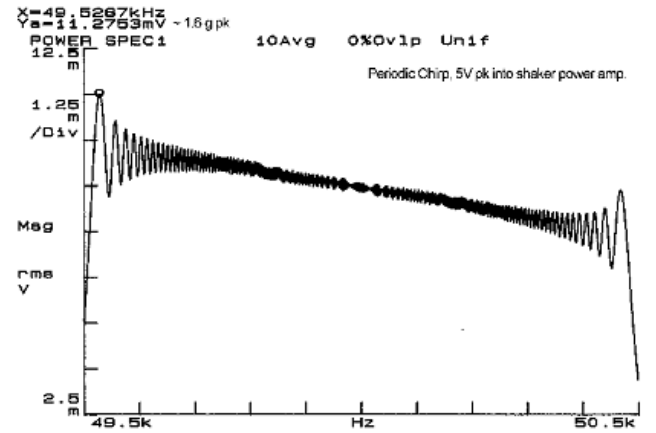

Reference 5V Periodic 51.6-52.6 kHz

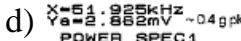

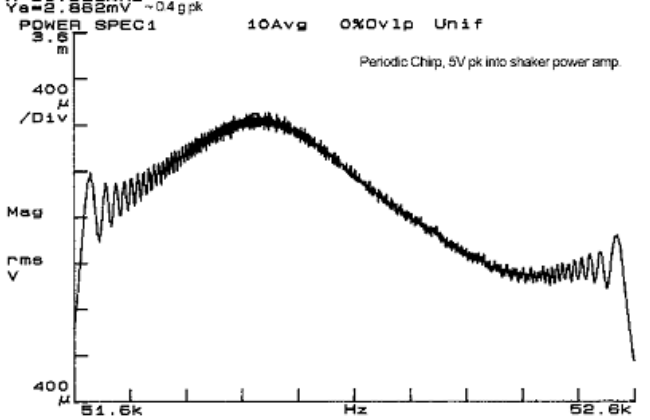

Reference 5V Periodic 39-40 kHz

e)

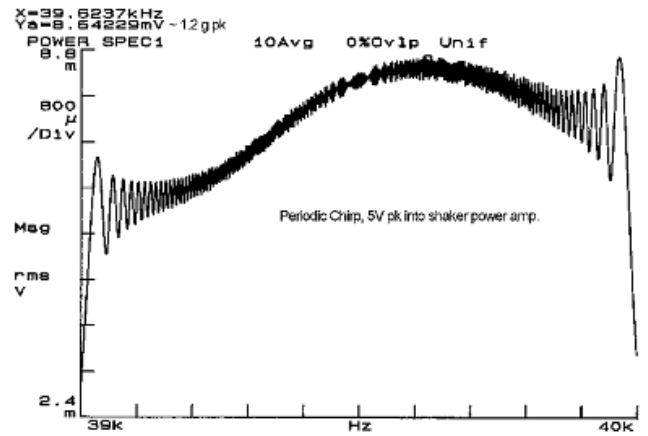

Figure 23. a) Signal response comparing baseline signal to three high periodic chirp noise cases for Accelerometer \#7 attached at 30 in-lbs torque at room temperature. b) Absolute value derivative of the signal responses. c) Noise band with peak acceleration at approximately $1.6 \mathrm{~g}$, d) $0.4 \mathrm{~g}$, and e) $1.2 \mathrm{~g}$.

The low and high chirp noise, generated in Figures 22 and 23 respectively, directly corresponds to the amount of noise signal amplitude at that noise bandwidth. When this noise signal drives the shaker system, the noise then appears in the diagnostic signal with the similar amplitude and bandwidth shown in the reference accelerometer. The result is a chirp noise that is not only shown in the diagnostic signal, but also the derivative magnitude. Even so, Figure 22b and 23b show that the derivative of the resonant frequency is greater than the derivative of the noise. Thus the SDA is still able to distinguish the resonant frequency from the periodic noise which is important for accelerometer health and attachment determination. 

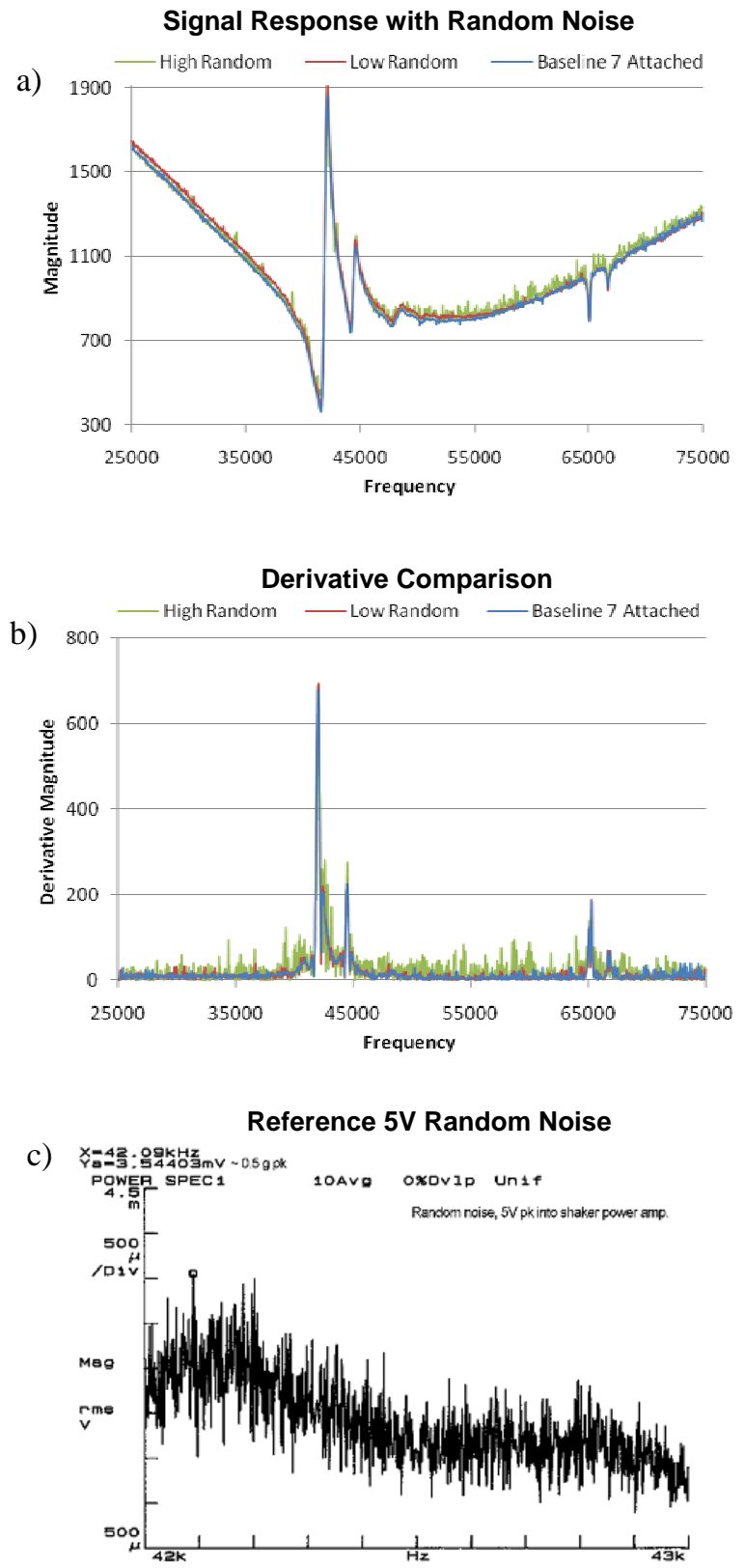

Figure 24. a) Signal response comparing baseline signal to two random noise cases for Accelerometer \#7 attached at 30 in-lbs torque at room temperature. b) Absolute value derivative of the signal responses. c) Noise band with peak acceleration at approximately $0.5 \mathrm{~g}$ for the $5 \mathrm{~V}$ high random and less than $0.5 \mathrm{~g}$ for the $1 \mathrm{~V}$ low random noise.

The random noise, generated in Figure 24, appears in the diagnostic signal just as the periodic noise did. Similarly, Figure 24b shows that the derivative of the resonant frequency is greater than the derivative of the noise. Thus the SDA is still able to distinguish the resonant frequency from the random noise which is important for accelerometer health and attachment determination.

\section{Temperature Investigation}

Calibration of the SDA system was done to compensate for frequency shift caused by changes in the temperature of an attached accelerometer. The largest frequency shift occurred between the temperature extremes, with $-73^{\circ} \mathrm{C}$ being the coldest and $+75^{\circ} \mathrm{C}$ being the warmest. Figure 25 shows the corresponding frequency shifts for some of these extremes. The largest frequency shift was approximately $1600 \mathrm{~Hz}$.

a)

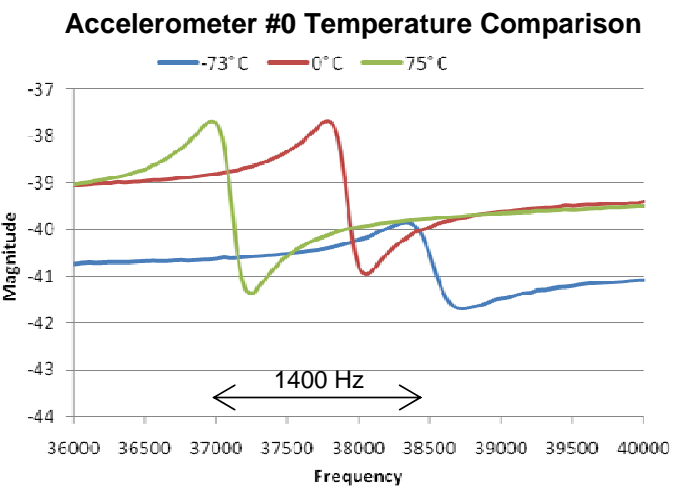

\section{Accelerometer \#2 Temperature Comparison}

b)

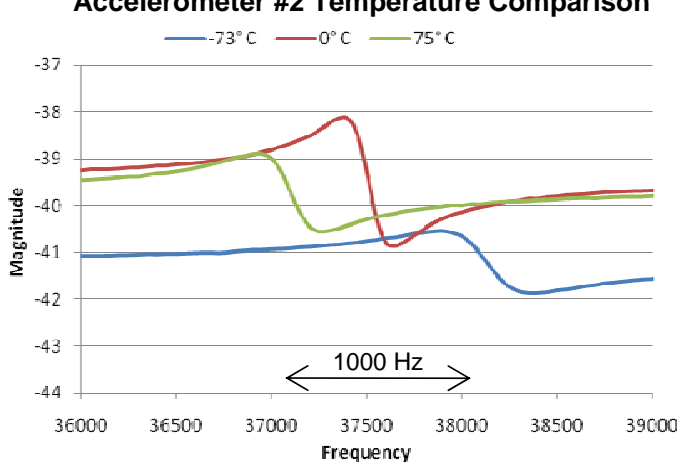

Accelerometer \#8 Temperature Comparison

c)

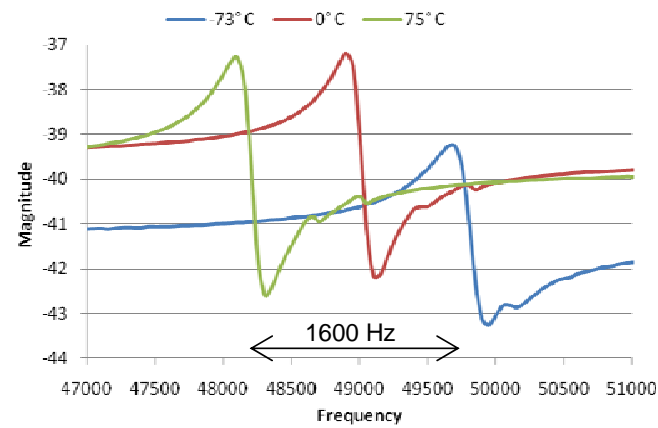

Figure 25. Resonant frequency shift from extreme temperature change for Accelerometer a) \#0, 1400 $\mathrm{Hz}, \mathrm{b})$ \#2, $1000 \mathrm{~Hz}$, and c) \#8, $1600 \mathrm{~Hz}$. 
D. Real Time Vibration and Temperature Tests

Testing of the attached and unattached condition with varying noise and temperature was done for the final SDA test in real time. It should be noted that a $100 \%$ reliability was attained in later post processing of the data by updating the SDA software algorithm accordingly.

The assessment parameters used in this test were determined from the Torque, Vibration, and Temperature Investigations. The following assessment parameters were used:

averaging range $=3$, derivative threshold $=100$, and resonant frequency correlation range $=5000$.

SDA Temperature Results

\begin{tabular}{|c|c|c|}
\hline & $\begin{array}{c}\text { Attached } \\
\mathbf{3 0} \text { in-lbs }\end{array}$ & $\begin{array}{c}\text { Unattached } \\
1 / 4 \text { Loose }\end{array}$ \\
\hline$-\mathbf{7 3}{ }^{\circ} \mathbf{C}$ & $35 \%^{*}$ & $85 \%^{*}$ \\
\hline$-\mathbf{5 0}{ }^{\circ} \mathbf{C}$ & $15 \%^{*}$ & $100 \%^{*}$ \\
\hline$-\mathbf{2 5}^{\circ} \mathbf{C}$ & $90 \%$ & $100 \%$ \\
\hline$+\mathbf{2 5}^{\circ} \mathbf{C}$ & $100 \% *$ & $100 \%^{*}$ \\
\hline $\mathbf{+ 7 5}^{\circ} \mathbf{C}$ & $100 \%$ & $100 \%$ \\
\hline$+\mathbf{1 0 0}^{\circ} \mathbf{C}$ & $100 \%$ & $100 \%$ \\
\hline
\end{tabular}

Table 1. SDA test results with varying temperature and attachment torque. These percentages are the number of successful real time SDA attachment determinations while tracking 2-4 resonant frequencies. Shaded area indicates temperatures where the accelerometers exhibited signal attenuation. * = Results Plotted.

SDA Vibration Results

\begin{tabular}{|c|c|c|}
\hline & $\begin{array}{c}\text { Attached } \\
\mathbf{3 0} \text { in-lbs } \\
+23^{\circ} \mathbf{C}\end{array}$ & $\begin{array}{c}\text { Unattached } \\
+23^{\circ} \mathbf{C}\end{array}$ \\
\hline $\begin{array}{c}\text { 0.5g 1V Perchirp 42- } \\
\text { 43 kHz }\end{array}$ & $100 \%$ & $100 \%$ \\
\hline $\begin{array}{c}\text { 0.3g 1V Perchirp } \\
\mathbf{4 9 . 5}-50.5 \text { kHz }\end{array}$ & $100 \%$ & $100 \%$ \\
\hline $\begin{array}{c}\text { 1.5g 5V Perchirp } \\
\text { 49.5-50.5 kHz }\end{array}$ & $100 \%$ & $100 \%$ \\
\hline $\begin{array}{c}\text { 0.4g 5V Perchirp } \\
\mathbf{5 1 . 6 - 5 2 . 6 ~ k H z}\end{array}$ & $100 \%$ & $100 \%$ \\
\hline $\begin{array}{c}\text { 1.2g 5V Perchirp 39- } \\
\text { 40 kHz }\end{array}$ & $100 \%$ & $100 \%$ \\
\hline$<0.5 g$ Low Random & $100 \%$ & $100 \%$ \\
\hline $\begin{array}{c}\text { 0.5g High 5V } \\
\text { Random }\end{array}$ & $100 \%$ & $100 \%$ \\
\hline
\end{tabular}

Table 2. SDA test matrix with varying noise and attachment torque. Shows number of successful SDA predictions for Accelerometer \#7 while tracking 1 resonant frequency at room temperature.
The diagnostic signals for the lower reliability conditions, $-73^{\circ} \mathrm{C}$ and $-50^{\circ} \mathrm{C}$ in Table 1 , are investigated further in Figures 26-35. In addition, the higher reliability $+25^{\circ} \mathrm{C}$ condition is plotted as the baseline reference. These plots are important in giving a visual representation of how the SDA processes a typical diagnostic signal, thus all ten accelerometers are presented. The annotations on the plots are: $\mathrm{T}=$ torqued 30 in-lbs (blue color plots), and $\mathrm{L}=1 / 4$ loose (orange color plots). The resonant frequencies monitored by the SDA to determine the real-time attachment for each specific accelerometer are circled in green with the circle width determined by the resonant frequency correlation range. The derivative threshold is shown with the black striped line. Clearly visible sources of error leading to an erroneous SDA fault, indicated in red, are signal attenuation, relative to the reference baseline, and ice formation at lower temperatures.

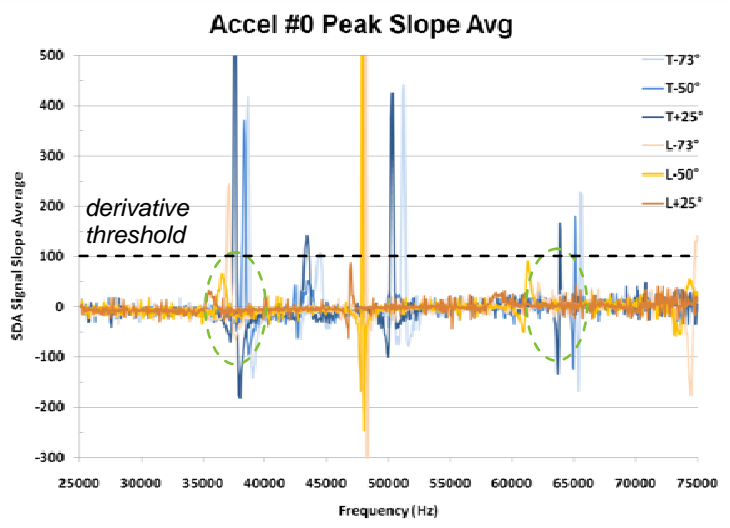

Figure 26. $\mathrm{L}-73^{\circ} \mathrm{C}$ and $\mathrm{L}-50^{\circ} \mathrm{C}$ show resonant frequencies that interfere with SDA processing, but these combined abnormalities are not enough to trigger an erroneous SDA fault given the baseline and assessment parameters chosen.

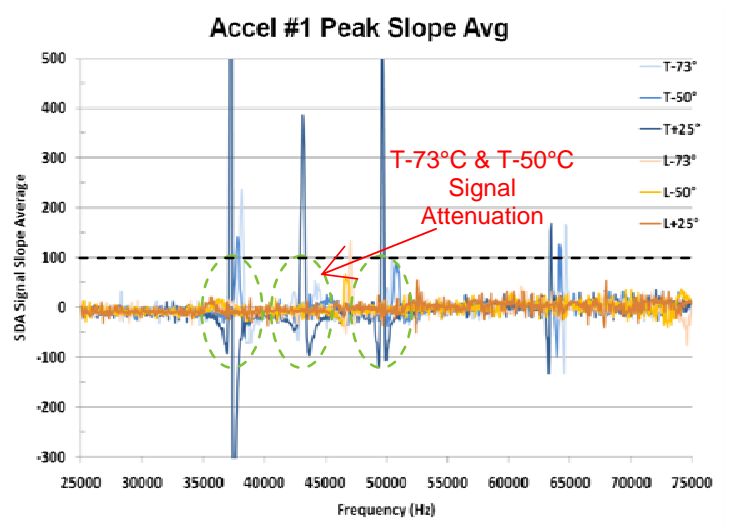

Figure 27. $\mathrm{T}-73^{\circ} \mathrm{C}$ and $\mathrm{T}-50^{\circ} \mathrm{C}$ show enough signal attenuation to drop the signal below the derivative threshold, triggering an erroneous SDA fault. 


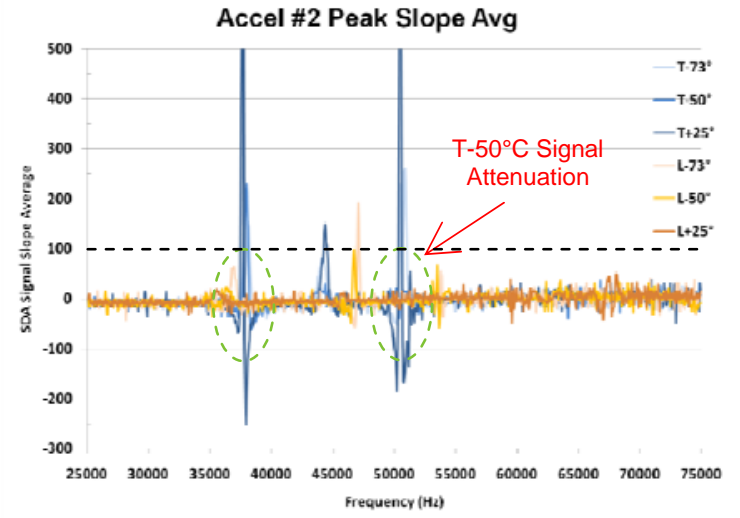

Figure 28. $\mathrm{T}-50^{\circ} \mathrm{C}$ shows enough signal attenuation to drop the signal below the derivative threshold, triggering an erroneous SDA fault.

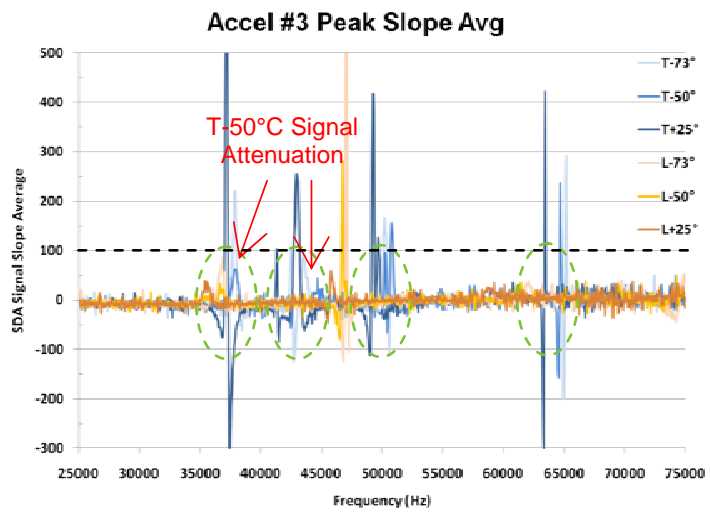

Figure 29. $\mathrm{T}-50^{\circ} \mathrm{C}$ shows enough signal attenuation to drop the signal below the derivative threshold, triggering an erroneous SDA fault.

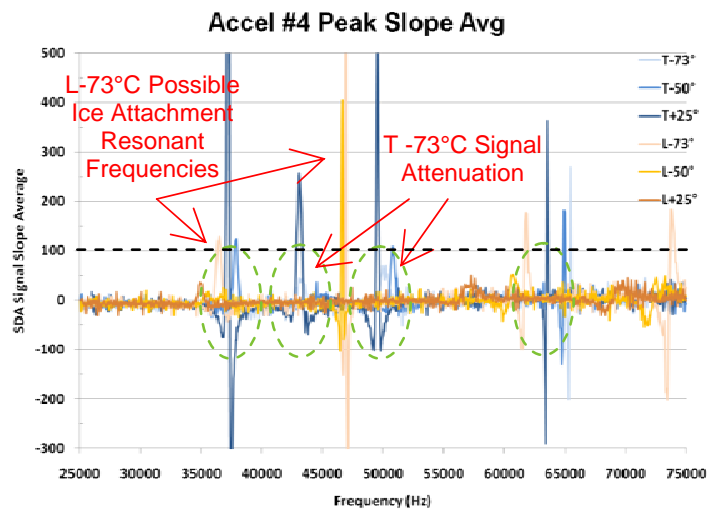

Figure 30. $\mathrm{T}-73^{\circ} \mathrm{C}$ shows enough signal attenuation to drop the signal below the derivative threshold, triggering an erroneous SDA fault. $\mathrm{L}-73^{\circ} \mathrm{C}$ shows resonant frequencies similar to an attachment condition, triggering an erroneous SDA fault.

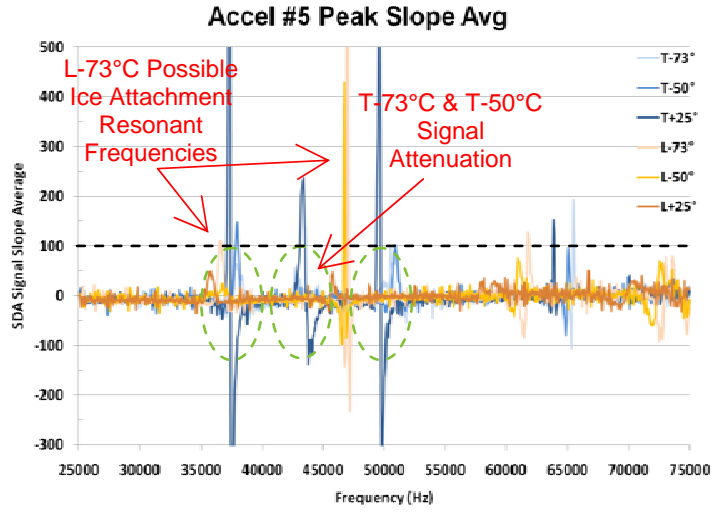

Figure 31. $\mathrm{T}-73^{\circ} \mathrm{C}$ and $\mathrm{T}-50^{\circ} \mathrm{C}$ show enough signal attenuation to drop the signal below the derivative threshold, triggering an erroneous SDA fault. $\mathrm{L}-73^{\circ} \mathrm{C}$ shows resonant frequencies similar to an attachment condition, triggering an erroneous SDA fault.

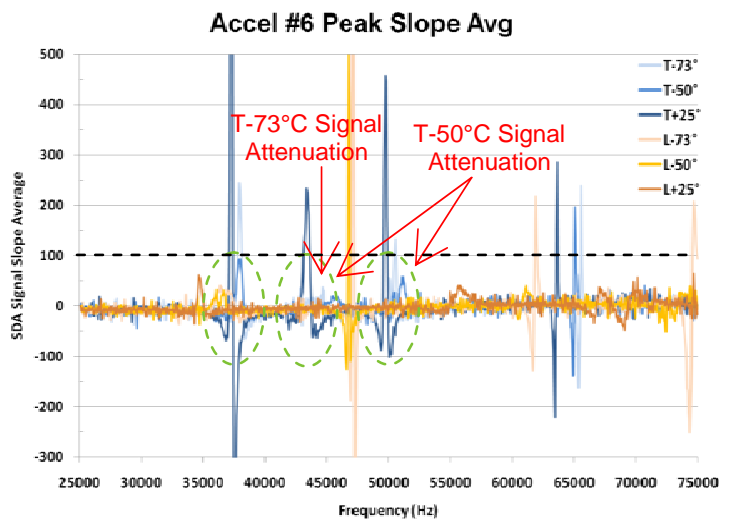

Figure 32. $\mathrm{T}-73^{\circ} \mathrm{C}$ and $\mathrm{T}-50^{\circ} \mathrm{C}$ show enough signal attenuation to drop the signal below the derivative threshold, triggering an erroneous SDA fault.

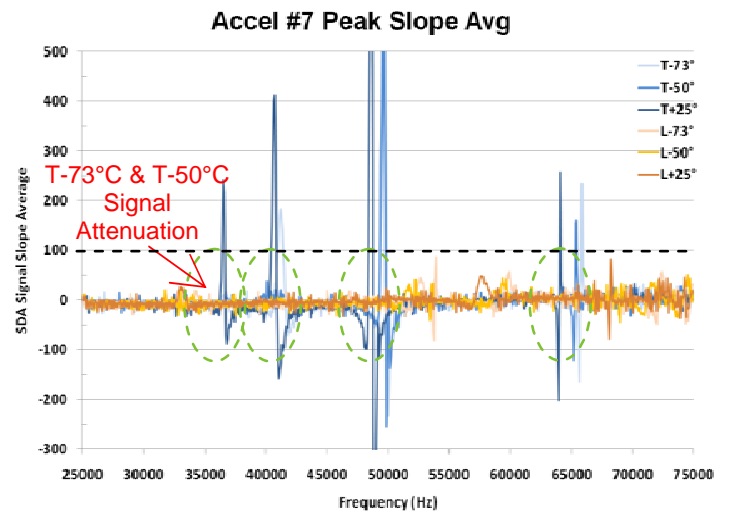

Figure 33. $\mathrm{T}-73^{\circ} \mathrm{C}$ and $\mathrm{T}-50^{\circ} \mathrm{C}$ show enough signal attenuation to drop the signal below the derivative threshold, triggering an erroneous SDA fault. 


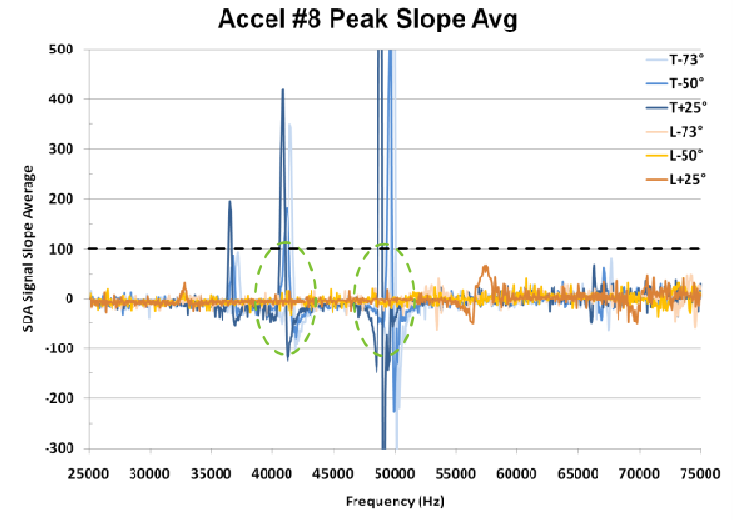

Figure 34. Diagnostic signals show little to none in signal attenuation and resonant frequency generation from the cold conditions as shown in the other figures.

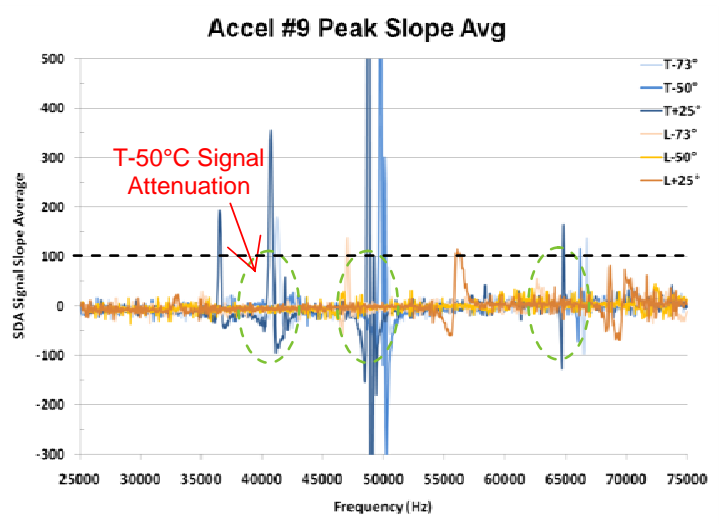

Figure 35. $\mathrm{T}-50^{\circ} \mathrm{C}$ shows enough signal attenuation to drop the signal below the derivative threshold, triggering an erroneous SDA fault.

In addition to the results from the temperature and vibration tests in Table 1 and 2, the SDA system was also successful in determining an open circuit. This was consistently repeated by simply disconnecting the cable to the accelerometer. The short circuit determination was also consistently correct, but was not tested extensively due to concerns in damaging the SDA system.

\section{Analysis and Discussion}

\section{A. Torque Investigation}

The torque tests investigated the effects of varying levels of torque on the diagnostic signal when determining the attachment condition. The three objectives included characterizing the diagnostic signal response resonant frequencies, investigating the attached/unattached condition, and compensating for resonance frequency shift from various torque levels of an attached accelerometer.
In characterizing the diagnostic signal response resonant frequencies, drastic changes were observed in the diagnostic signal response when the accelerometer was changed from the attached to unattached condition, as shown in Figures 18 to 19. The drastic change in the diagnostic signal response included a change in the quantity, location, and structure of its resonant frequencies. These changes give support for the use of a resonant frequency matching technique to determine accelerometer attachment.

When investigating the attached/unattached condition, it was determined that the 10 in-lbs would be most suitable for the lower torqued extreme in determining the attached condition. Gradually increasing the torque from a loose condition, to just touching, to tighter, then finally to maximum finger tightness generally repeated the resonant frequency shift pattern consistent with higher level torque values, such as $10,20,30$, and 40 in-lbs. Subtle changes in the contact surface are very sensitive to introducing resonant frequencies. The loose and just touching condition were more unpredictable, such as with localizing the resonant frequency peaks, than when compared to the higher torque conditions of maximum finger tightness and 10 in-lbs. Because the 10 in-lbs is a quantitatively measureable value and because it is stable, it makes for a suitable lower attached torque extreme. Accordingly, the 10 in-lbs torque effects are taken into account when calibrating the SDA system, specifically with the resonant frequency correlation range. Also, when determining the baseline attachment torque, 30 in-lbs was chosen because it is well past the stable 10 in-lbs torque attachment, thus ensuring quality resonant frequency baseline signals.

The SDA system was calibrated to compensate for frequency shift during changes in the torque of an attached accelerometer. The results of the torque tests showed that approximately $500 \mathrm{~Hz}$ would be large enough to compensate for the mounting torque shift in the resonant frequency. This value was determined by taking the largest frequency shift from the mounted torque change. In the Torque Investigation, $500 \mathrm{~Hz}$ was the largest frequency shift which is shown in Figure 21a. This $500 \mathrm{~Hz}$ torque compensation value is used in addition to the temperature compensation value for calculating the resonant frequency correlation range.

\section{B. Vibration Investigation}

The vibration tests investigated noisy signal responses that can often lead to falsely flagged conditions if the noise is greater than the derivative 
threshold assessment parameter. The SDA system handles high levels of noise by signal averaging and using an effective derivative threshold for resonant frequency localization. This allowed for accurate attached and unattached determination. However, care must be taken so that averaging does not wash out the resonant frequencies from the signal response. For this reason, the averaging range was set to do a moving average of only 3 data points which was low enough to not significantly smooth the resonant frequencies.

The best derivative threshold is one that will pass through the derivative of the resonant frequency, but not through the derivative of the signal noise spikes. Doing so reveals that the derivative threshold of 50 to 100 worked well with the averaging range set to 3 for monitoring multiple resonant frequencies. A larger derivative threshold can be used to reduce the effect of noise, but this would also result in a reduction in the number of resonant frequencies monitored when those resonant frequency peaks are below the derivative threshold. A reduction in the number of resonant frequencies monitored decreases the SDA system's ability to monitor for changes in the diagnostic signal. Thus a balance between the number of resonant frequencies monitored and using a large enough derivative threshold must be attained.

The prototype SDA system utilized a moving average to keep processing time to a minimum. While smoothing random noise spikes, this also had the negative side effect of reducing the resonance peaks that are monitored by the SDA software algorithm. Future generations of a faster processing SDA system will utilize time averaging to reduce noise while maintaining the resonant frequency strength.

As stated previously, the attachment must be ensured for true vibrations to be sensed by the accelerometer. This was evident in the loose condition noise testing. None of the accelerometers were able to pick up any noise while in the loose state. The diagnostic responses were the same as the loose states without the noise. Thus the loose conditions were not plotted for the noise testing.

Care had to be taken in selecting an appropriate periodic chirp noise. If the noise bandwidth was strong enough and sufficiently overlapped the internal resonant frequencies of the accelerometer or attachment, then this frequency would show up periodically as the SDA is swept across the $25 \mathrm{kHz}$ to $75 \mathrm{kHz}$ range. As shown in Figure 23a, the noise generated for the $5 \mathrm{~V}$ periodic chirp $39-40 \mathrm{kHz}$ partly overlaps the resonant frequency of the accelerometer/attachment baseline. This causes periodic bumps to appear outside the normal bandwidth of the noise. In this specific case, the periodic bump was not large enough to interfere with the operation of the SDA as is apparent from inspecting the derivative data in Figure 23b. The derivative of the noise bump is smaller than the derivative of the resonant frequency of the baseline diagnostic signal, thus allowing the SDA to ignore the noise bumps altogether. In other cases, it was possible to completely overwhelm the diagnostic data with the noise. It is believed that normal accelerometer operating conditions will not involve periodic noise, but rather random noise as shown in Figure 24. Also, there are future hardware improvements to the SDA, specifically with the use of a high speed digital signal processor, which may address this scanning issue.

When testing with the shaker system, the SDA diagnostic baselines had to be regenerated because the accelerometers were mounted on a new shaking base which was different than the previously mounted masses used in the temperature tests. The new baselines resulted in new resonant frequencies being tracked by our SDA software algorithm.

\section{Temperature Investigation}

The temperature tests investigated the resonant frequency shift in the signal response from temperature change. The SDA system compensated for the resonant frequency shift by using a range that includes any frequency shift up to and including the maximum shift caused by temperature change. The results of the temperature tests showed that approximately $1600 \mathrm{~Hz}$ was large enough to compensate for the temperature change shift in the resonant frequency. This value was determined by taking the largest frequency shift from temperature change. In the Temperature Investigation, $1600 \mathrm{~Hz}$ was the largest frequency shift which is shown in Figure 25c. This $1600 \mathrm{~Hz}$ temperature compensation value is used in addition to the previously calculated torque compensation value for calculating the resonant frequency correlation range. The resonant frequency correlation range assessment parameter compensates for the worst case torque and temperature change to help correctly match resonant frequencies. Summing the calculated torque and temperature compensation values yields $2.1 \mathrm{kHz}$ as the maximum allowable difference between two resonant frequencies. An additional $400 \mathrm{~Hz}$ was then added for error tolerance purposes to get $2.5 \mathrm{kHz}$. To convert this difference value to a range, it is doubled. Thus the resonant frequency correlation range $=5$ $\mathrm{kHz}$. Put another way, the doubling occurs because 
the resonant frequency correlation range includes 3 $\mathrm{kHz}$ less than and greater than the inspected frequency. The resonant frequency correlation range can be further customized to isolate and/or ignore specific resonant frequencies.

\section{Vibration and Temperature Tests}

The SDA system has successfully demonstrated the ability to monitor the attached and unattached condition while compensating for temperature changes and mitigating the negative effects of noise.

Temperature testing revealed issues with the colder temperatures, specifically the $-75^{\circ} \mathrm{C}$ and $-50^{\circ} \mathrm{C}$ range, as shown in Figures 26-35. Analyzing the SDA diagnostic data revealed a signal attenuation, or loss of signal amplitude. It is believed this is caused by a temperature and capacitance dependency. The lower resonance amplitudes from the signal attenuation resulted in an inability to correctly identify resonant frequency locations. This often resulted in a false positive, reporting an untorqued condition when in fact the SDA was torqued. This is the reason for the $35 \%$, 15\%, and $90 \%$ reliability in Table 1 . To correct for this, instead of tracking multiple resonances, in this report the SDA tracked 2-4, the SDA system could focus on the single largest resonant frequency. This would be similar to using a higher derivative threshold such as switching from the derivative threshold 1 to derivative threshold 2 in Figure 9. It should be noted that focusing on just one resonant frequency increases the likelihood that the loose condition also has that same resonant frequency, which could result in the SDA reporting an attached condition when in fact it is unattached. Another issue with the cold temperatures is ice; specifically with the $-73^{\circ} \mathrm{C}$ untorqued condition. This is the reason for the $85 \%$ reliability shown in the Table 1 . Ice forming between the accelerometer and base could result in a tighter fit of the attachment. This is similar to the effect of torquing the SDA, causing it to appear attached. Similar resonant frequencies to the baseline are generated when the accelerometer is attached by ice resulting in the erroneous SDA fault. To reduce this source of error while testing, the investigator can remove excess water vapor, ensure ice is removed and/or broken up, or use a looser mount so that the accelerometer and base are farther from each other to reduce the ability for the ice to form an attachment between them.

The SDA system was also capable of monitoring for open and short circuits. The SDA drive signal is a current source. It drives a circuit of any impedance with a fixed current. When the SDA sees a zero resistance, ground, the voltage is zero. When the
SDA sees infinite impedance, open circuit, the voltage goes to the high rail. Thus the software can monitor for these changes and issue a fault accordingly.

\section{Conclusion}

SDA testing on 10 accelerometers included Torque, Vibration, and Temperature Investigations. Findings from these investigations guided the development of the SDA system processing software. Sources of error were explored in detail. Assessment parameters such as noise averaging, derivative thresholds, and resonant frequency correlation were used to determine the attached and unattached fault conditions of the SDA system. Changes in vibrations, temperatures, and states of attachment were tested. This allowed for further refinement of the software for real time determination of the SDA system health and attachment status. The real time test results indicate a reliable prototype SDA system for temperatures $+25^{\circ} \mathrm{C}$ to $+100^{\circ} \mathrm{C}$ and noise vibrations from $0 \mathrm{~g}$ to $1.5 \mathrm{~g}$. Further post processing of the data provided for SDA system reliability beyond these temperature and noise ranges.

Future work includes updating the SDA system hardware to a faster frequency scanner. A faster frequency scanner would allow for more accelerometer health polling. This would allow for faster SDA response as well as making the use a multiple scan signal average more practical, which would be superior to the moving average currently used to reduce signal noise. Further software refinements and features are forthcoming such as an automated ability to easily distinguish and load baseline resonant frequencies for a newly attached SDA.

\section{References}

1 Davidson, M., and Stephens, J., "Advanced Health Management System for the Space Shuttle Main Engine”, 40th AIAA/ASME/SAE/ASEE Joint Propulsion Conference, Fort Lauderdale, Florida, July 11-14, 2004.

${ }^{2}$ Simon, D., Garg, S., Hunter, G., Guo, T.-H., and Semega, K.,"Sensor Needs for Control and Health Management of Intelligent Aircraft Engines”, NASA/TM-2004-213202.

${ }^{3}$ Atherton, W. J. and Flanagan, P. M., " A Self Diagnostic System for Piezoelectric Sensors ", Proceedings of 25th AIAA/ASME/SAE/ASEE Joint Propulsion Conference, Monterey, Ca., July 10-12, 1989

${ }^{4}$ Flanagan, P. M and Atherton, W. J., "Developing a SelfDiagnostic System for Piezoelectric Sensors", Proceedings of 26th AIAA/ASME/SAE/ASEE Joint Propulsion Conference, Orlando, Fl., July 16-18, 1990 
${ }^{5}$ Flanagan, P. M., "In-Situ Measurement of Sensitivity for a Piezoelectric Sensor", Proceedings of 27th AIAA/ASME/SAE/ASEE Joint Propulsion Conference, Sacramento, Ca., June 24-26, 1991

${ }^{6}$ Flanagan, P. M., "Design of a Self-Diagnostic Beam-Mode Piezoelectric Accelerometer", Proceedings of 28th AIAA/ASME/SAE/ASEE Joint Propulsion Conference, Nashville, Tenn., July 6-8, 1992

${ }^{7}$ Flanagan, P. M., "Dedicated System for a Self-Diagnostic Piezoelectric Accelerometer", Proceedings of 30th AIAA/ASME/SAE/ASEE Joint Propulsion Conference, Indianapolis, In., June 27-29, 1994

${ }^{8}$ Flanagan, P. M., "Design of a Self-Compensation System for Piezoelectric Accelerometers", Proceedings of 32nd AIAA/ASME/SAE/ASEE Joint Propulsion Conference, Lake Buena Vista, Fl., July 1-3, 1996

${ }^{9}$ Flanagan, P. M., Lekki, J., 'A Self-Diagnostic System for the M6 Accelerometer', Proceedings of 37th AIAA/ASME/SAE/ASEE Joint Propulsion Conference, Sault Lake City, Utah 2001.

${ }^{10}$ Jiang, Q., Cross, L., "Effects of porosity on electric fatigue behaviour in PLZT and PZT ferroelectric ceramics”, J. of Mat. Sci., Vol. 28, 1993, pp. 4536-4543.

${ }^{11} \mathrm{Kim}$, S., Jiang, Q., "Microcracking and electric fatigue of polycrystalline ferroelectric ceramics”, Smart Mater. Struct., Vol. 5, 1996, pp. 321-326.

${ }^{12}$ Gao, H., Zhang, T., Tong, P., "Local and global energy release rates for an electrically yielded crack in a piezoelectric ceramic”, J. Mech. Phys. Solids, Vol. 45, No. 4, 1997, pp. 491-510.

${ }^{13}$ Shen, S., Nishioka, T., "Fracture of piezoelectric materials: energy density criterion”, Theorectical and Applied Fracture Mechanics, Vol. 33, 2000, pp. 57-65.

${ }^{14}$ Wang, H., Singh, R., "Crack propagation in piezoelectric ceramics: Effects of applied electric fields”, J. Appl. Phys., Vol. 81, No. 11, 1997, pp. 7471-7479.

${ }^{15}$ Lekki, J., Tokars, R., Jaros, D., Riggs, T., Evans, K., Gyekenyesi, A., "Self Diagnostic Accelerometer for mission critical health monitoring of aircraft and spacecraft engines”, 47 $7^{\text {th }}$ AIAA Aerospace Sciences Meeting, Orlando, FL, January 2009. 